\title{
Monoclonal Gammopathy of Undetermined Significance and Risk of Progression
}

\author{
Nahla AM Hamed* \\ Professor of Hematology, Alexandria University, Egypt \\ Submission: February 06, 2017; Published: February 21, 2017 \\ "Correspondence Address: Nahla AM Hamed, Professor of Hematology, Alexandria University, Egypt
}

Abstract

The rate of progression of MGUS to MM is $0.5-1 \%$ per year and to lymphoproliferative disorder is $1 \%$ per year. Even after $>25$ years of follow-up, the risk of progression does not decrease. Patients with MM and prior knowledge of MGUS had better survival, suggesting that earlier treatment of MM leads to better survival. miR-221/222 in serum and plasma cells may be biomarkers for early diagnosis of MGUS.

Abbreviations: MGUS: Monoclonal Gammopathy of Undetermined Significance; FLC: Free Light Chain; BM: Bone Marrow; MM: Multiple Myeloma; SPEP or UPEP: Serum or Urine Protein Cellulose Acetate Electrophoresis; SIEP: Serum Immuno Electro-Phoresis; IFE: ImmunoFixation Electrophoresis; MAG: anti-Myelin-Associated Glycoprotein; SMM: Smoldering Multiple Myeloma; GEP: Gene Expression Profiling.

\section{Introduction}

MGUS is an asymptomatic premalignant plasma cell disorder occurring mainly in the elderly population [1]. MGUS is present in roughly $3-4 \%$ of the population over the age of 50 years. Almost all patients have IgG or IgA MGUS [2].

\section{Definitions}

i. Non-IgM MGUS is defined by a serum M-protein $<30$ $\mathrm{g} / \mathrm{L}$, clonal bone marrow plasma cells percentage $<10 \%$ and absence of end-organ damage such as hypercalcemia, renal insufficiency, anemia, and bone lesions (CRAB) or amyloidosis that can be attributed to plasma cell proliferative disorder [3].

ii. IgM MGUS is defined by serum IgM M-protein $<30 \mathrm{~g} / \mathrm{L}$ and bone marrow lymphoplasmacytic infiltration $<10 \%$. No evidence of anemia, constitutional symptoms, hyper viscosity, lymphadenopathy, hepatosplenomegaly, or other end-organ damage that can be attributed to the underlying lymph proliferative disorder [3].

iii. Light-chain MGUS is defined by abnormal FLC ratio $(<0.26$ or $>1.65)$ and increased level of the appropriate involved light chain (increased k FLC in patients with ratio $>1.65$ and increased $\mathrm{k}$ FLC in patients with ratio $<0.26$ ), no immunoglobulin heavy-chain expression on immunofixation, clonal bone marrow plasma cells $<10 \%$, urinary monoclonal protein $<500 \mathrm{mg} / 24 \mathrm{hr}$ and absence of end-organ damage such as hypercalcemia, renal insufficiency, anemia, and bone lesions (CRAB) or amyloidosis that can be attributed to plasma cell proliferative disorder [3].
In persons aged 50 years and older, light-chain MGUS has a prevalence of $\sim 0.7-0.8 \%$ [2]. Importantly, it is possible that a small proportion of patients with apparent light-chain MGUS do not have a true clonal disorder but rather renal dysfunction or polyclonal activation. In the latter condition, there are increased levels of $\gamma$ and $\mathrm{k}$ chains with a normal ratio [2].

\section{Etiology}

Obesity, black race [4] and personal history of autoimmune diseases, inflammatory conditions, and infections are associated with increased risk of MGUS [2]. Inflammation in the setting of autoimmunity may serve as a trigger for MGUS. In addition, a common genetic susceptibility for developing both an autoimmune disease and MGUS might also exist [5]. Occupational studies have demonstrated that exposure to radiation or pesticide is associated with the development of MGUS. Vietnam War veterans exposed to the herbicide Agent Orange had a 2.4fold increased risk for MGUS [2].

\section{MGUS and risk of non-malignant disorders}

The importance of MGUS not only lies in the increased risk of developing a hematologic malignancy but the small clone may also be responsible for severe organ damage through the production of a toxic M-protein which has an antibody activity or deposits in tissues. In addition, the pre-malignant clone induced alterations in the BM microenvironment resulting [6] in an increased risk of venous and arterial thrombosis, lifetime risk of infection and osteoporosis [7]. Several studies report an 
approximately 2-3 fold increased risk of developing deep venous thrombosis or pulmonary embolism [6]. Hypothyroidism was a common finding, compared to the general population [1].

Peripheral neuropathy is often seen in association with monoclonal gammopathies. The pathogenesis is thought to be related to the effect of the monoclonal protein (or secondary biological mechanisms) on the peripheral nerves, and can occur in patients with MGUS without need for malignant transformation. Thus, peripheral neuropathy alone is not a myeloma-defining event [3]. In patients with neuropathy, particularly demyelinating neuropathies, the para-protein associated with MGUS is frequently IgM. The para-proteins are usually kappa light chains. Approximately half of patients with IgM MGUS have anti-MAG antibodies. They are typically older males, in the 6th to 9 th decades of life. The characteristic clinical picture is an insidiously progressive, distal, predominantly sensory neuropathy, with mild distal weakness. Large fibers controlling vibration and proprioception are affected more than pain and temperature sensation, resulting in progressive balance problems [8].

\section{MGUS and risk of malignant disorders}

MGUS patients have an approximately 1.5 fold increased risk of developing a non hematologic malignancy. First degree relatives of patients with MGUS have approximately $10 \%$ increased risk of developing solid tumors. Furthermore, patients with MGUS appear to have a 2-8 fold increased risk of developing myeloid malignancies including myelodysplastic syndromes, acute myeloid leukemia and polycythemia vera. MGUS is a precursor condition to multiple myeloma and other lymphoproliferative disorders [6]. The rate of progression of MGUS to MM is $0.5-1 \%$ per year and to lymphoproliferative disorder is $1 \%$ per year [3]. Even after $>25$ years of follow-up, the risk of progression does not decrease [2].

\section{Progression of MGUS to multiple myeloma}

About $80 \%$ of MM originates from non-Ig MMGUS and $20 \%$ from light-chain immunoglobulin MGUS. IgM MGUS usually evolves into Waldenstrommacroglobulinemia or other lymphoproliferative disorders, but in rare instances Ig MMGUS can progress to MM (IgM myeloma). The risk of progression for light-chain MGUS is lower when compared to the risk of progression in conventional MGUS. Patients with asymptomatic light chain monoclonal gammopathy are at risk of developing light-chain $\mathrm{MM}$ or immunoglobulin light-chain amyloidosis $\mathrm{AL}$ amyloidosis) [3].

MGUS is a genetically advanced lesion, wherein tumor cells carry many of the genetic changes found in MM cells [9]. Analysis of populations at increased risk of MGUS suggests the possible existence of polyclonal phase preceding the development of MGUS to MM [9]. In patients with MGUS, a substantial proportion of polyclonal plasma cells persist, whereas in multiple myeloma almost all plasma cells (>95\%) are clonal [3]. Recent advances in mouse modeling of MGUS suggest that the clinical dormancy of the clone may be regulated in part by growth controls extrinsic to the tumor cells. Interactions of MGUS with immune cells, bone cells and others in bone marrow niche may be regulators of malignant transformation. These interactions involve a bidirectional cross-talk leading to both growth-supporting and inhibitory signals [9]. Progression from MGUS to SMM and then to $\mathrm{MM}$ is also mediated via competition between subclones and outgrowth of the fittest [2]. Patients with MM and prior knowledge of MGUS had better survival, suggesting that earlier treatment of MM leads to better survival [10].

\section{Diagnosis}

Presence of para-protein can be detected in urine or serum when patients are screened with SPEP or UPEP. SIEP and IFE are even more sensitive measures that can verify the monoclonal nature of the para-protein. Half of the patients with IgM gammopathy and peripheral neuropathy have anti-MAG antibodies, by western blot orenzyme-linked immunosorbent assay [8]. miR-221/222 in serum and plasma cells may be biomarkers for early diagnosis of MGUS [11].

\section{Predictors of malignant transformation in MGUS}

Most guidelines recommend annual follow up with laboratory tests until life expectancy becomes limited [12]. Various presenting features are helpful in predicting risk of progression of MGUS to symptomatic disease and therefore in individualizing follow-up [2]. The size of the MGUS clone as determined by BM plasma cell percentage or M-protein level is an important risk factor for progression of MGUS [2]. Other identified risk factor is low serum albumin [13]. However, percentage of marrow plasmacytosisis subjected to potential sampling bios related to focal lesion suggesting the importance of tumor biology in addition to tumor bulk [14].

Several biological characteristics of the clone have predictive value in conventional MGUS including heavy-chain isotype (IgA/ $\operatorname{IgM}>\operatorname{IgG}$ ); light-chain production as determined by abnormal serum FLC ratio or presence of Bence-Jones proteinuria; detection of circulating plasma cells or clonal B cells; increased bone resorption in bone biopsy; clonal heterogeneity; DNA aneuploidy by flow cytometry; and abnormal metaphase cytogenetics. GEP of purified plasma cells has recently been demonstrated to have prognostic value [2]. GEP of purified tumor cells revealed that all molecular subtypes of clinical myeloma are also represented in the asymptomatic monoclonal gammopathy phase [14].

Furthermore, suppression of nonclonal BM plasma cells, based on multiparameter flow cytometric analysis, has been identified as a risk factor for progression, which explains the predictive value of reduced levels of polyclonal serum immunoglobulins. Detection of focal lesions by MRI at baseline and development of focal lesions by MRI or PET-CT are predictive for progression to active MM. It is currently unknown whether 


\section{Cancer Therapy \& Oncology International Journal}

specific chromosomal abnormalities, including del (17p) and $\mathrm{t}[4,14]$, are predictive of malignant progression in MGUS [2]. No predictive factors for progression have been identified for light-chain MGUS. It is currently unknown whether higher levels of the involved light chain result in a higher risk of malignant transformation in light-chain MGUS [2].

\section{Conclusion}

MGUS was associated with numerous malignant and nonmalignant disorders [1]. Baseline characteristics and the dynamics of the plasma cell clone during the first years of follow-up are helpful in predicting the risk of transformation to a malignant plasma cell disorder [2].

\section{References}

1. Maltezas D, Eftychia N, Papaioannou P, BitsaniA, Tzenou T, et al. (2015) Study of MGUS-Series: disease evolution, coexistent disorders and other clinical observations. Blood 126: 5323.

2. Van de Donk NWCJ, Mutis T, Poddighe PJ, Lokhorst HM, Zweegman S (2016) Diagnosis, risk stratification and management of monoclonal gammopathy of undetermined significance and smoldering multiple myeloma. Int J Lab Hemtol 38 (Suppl 1): 110-122.

3. Rajkumar SV, Dimopoulos MA, Palumbo A, Blade J, Merlini G, et al (2014) International Myeloma Working Group updated criteria for the diagnosis of multiple myeloma. Lancet Oncol 15(12): e538-548.

4. Chang SH, Luo S, Thomas TS, O’Brian KK, Coldotz GA, et al. (2016) Obesity and the transformation of monoclonal gammopathy of undetermined significance to multiple myeloma: a population based cohort study. J Natl Cancer Inst 109(5).

5. Shimanovsky A, Alvarez Argote J, Murali S, Dasanu CA (2016) Autoimmune manifestations in patients with multiple myeloma and

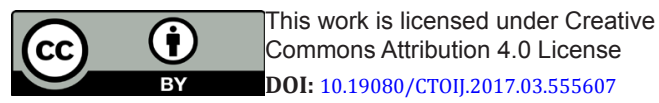

monoclonal gammopathy of undetermined significance. BBA Clin 6: 12-18.

6. Van de Donk NW, Palumbo A, Johensen HE, Engelhardt M, Gay F, et al. (2014) The clinical relevance and management of monoclonal gammopathy of undetermined significance and related disorders: recommendations from the European Myeloma Network. Haematologica 99(6): 984-996.

7. Goebel TE, Schltz NK, Pillai AC, Caimi P, Koroukian SM, et al. (2014) Effects of monoclonal gammopathy of undetermined significance on outcomes after solid organ transplant. Blood 124: 3507.

8. Ramchandren S, Lewis RA (2012) An update on monoclonal gammopathy and neuropathy. Curr Neurol Neurosci Rep 12(1): 102110.

9. Dhodapkar MV (2016) MGUS to myeloma: a mysterious gammopathy of underexplored significance. Blood 128: 2599-2606.

10. Sigurdardottir EE, Turesson I, Lund SH, Lindqvist EK, Mailankody S, et al. (2015) The role of diagnosis and clinical follow-up of monoclonal gammopathy of undetermined significance on survival in multiple myeloma. JAMA Oncol 1(2): 168-174.

11. Yang S, Wang W, Jin H, Zhong Y, Xie X (2016) Expression of microRNA-221/222 in patients with monoclonal gammopathy of undetermined significance and multiple myeloma. Zhejiang Da Xue Xue Bao Yi Xue Ban 45(4): 371-378.

12. Go RS, Heien HC, Sangaralingham LR, Habermann EB, Shah ND (2016) Monoclonal gammopathy of undetermined significance (MGUS) follow up practice patterns: frequency of visits, types of tests and concordance with clinical practice guidelines. Blood 128: 5906.

13. Lindqvist E, Lund SH, Costello R, Burton D, Korde NS, et al. (2016) Risk of progression in monoclonal gammopathy of undetermined significance (MGUS): results from a population based screening study. Blood 128: 5650.

14. Dhodapkar MV, Sexton R, Waheed S, Usmani S, Papanikolaou X, et 